\title{
COMMENTARIES
}

\section{Human Endotoxin Administration as an Experimental Model in Drug Development}

\author{
AF Suffredini $^{1}$ and RJ Noveck ${ }^{2}$
}

\begin{abstract}
Linking human physiology to inflammatory mechanisms discovered in vitro or in animal models is essential to determine their importance. Innate immunity underlies many of these inflammatory responses in health and disease. Bacterial endotoxin is the quintessential trigger of innate immune responses. When administered to humans, endotoxin has been an important means of demonstrating key inflammatory mechanisms in vivo. Furthermore, endotoxin challenges have provided opportunities to test the effects of novel inflammation-modifying agents in humans.
\end{abstract}

Gram-negative bacterial lipopolysaccharide, or endotoxin, has been administered to humans for more than 100 years under a variety of circumstances. Its use has come in and out of favor at various stages of medical knowledge-as a therapeutic, a diagnostic tool, or a means to study human inflammation. During the first half of the twentieth century, therapeutic fever induced by bacterial components was used to treat a variety of chronic infections. In 1942, patients with neurosyphilis were treated with a purified endotoxin preparation derived from Salmonella. ${ }^{1}$ In parallel with its use in fever therapy, killed Gram-negative bacteria were found to have anti-tumor effects, and several preparations were marketed commercially for this purpose until 1950. ${ }^{1}$ Because of the predictable systemic responses, endotoxin was used to assess pituitary reserves and became an important tool with which to study the pathogenesis of fever and bone marrow reserves.

In 1976, a large quantity of endotoxin was extracted from Escherichia coli O:113:H10:K negative under the direction of National Institute of Allergy and Infectious Diseases and the US Food and Drug Administration to provide a "national biological reference standard to be made available to pharmaceutical manufacturers and qualified biomedical investigators as an aid to standardization of bioassays and research with endotoxin."2 Since then, this endotoxin preparation has been used in many human challenge studies around the world. It remains the World Health Organization standard for endotoxin assays used in the pharmaceutical indus- try. ${ }^{3}$ Some regulatory authorities currently require that the endotoxin used in human challenge studies be good manufacturing practice grade endotoxin, and this is of limited availability worldwide. We describe an overview of human endotoxin challenge studies. More detailed information is summarized in Tables 1 and $\mathbf{2}$ (and in the tables' references in the Supplementary References online).

Before the 1980s, the innate immune system was considered a relatively primitive and nonspecific means of initiating adaptive immunity. However, the discovery of Toll-like receptors and the characterization of key early inflammatory mediators (e.g., tumor necrosis factor, interleukin-1) stimulated an explosion of research that began to define the unique features and complexity of innate immunity as a highly conserved defense strategy that protects multicellular organisms from infection. ${ }^{4}$ Within minutes of ligandreceptor binding of microbial molecular structures, myeloid and lymphoid cells as well as humoral proteins are activated. The resultant cascade of inflammatory events recruits inflammatory cells and proteins to the nidus of infection, killing the microbe and initiating repair of damaged tissue. Adaptive immune responses are facilitated by these responses, resulting in long-term highly specific immunity to the microbe. Because of its central role in many aspects of innate immunity and, specifically, some infectious diseases, endotoxin challenges emerged as a means to study inflammation in humans. Human exposure to endotoxin leads to sterile inflammation, with many features qualitatively similar to those of a true infection with the advantages that it is safe, reproducible, and of limited duration (Tables 1 and 2). It has provided a proof of principle for inflammation-modifying interven-

\footnotetext{
${ }^{1}$ Critical Care Medicine Department, Clinical Center, National Institutes of Health, Bethesda, Maryland, USA; ${ }^{2}$ Department of Medicine, Division of Clinical Pharmacology, Duke Clinical Research Unit, Duke University School of Medicine, Durham, North Carolina, USA. Correspondence: RJ Noveck (robert.noveck@duke.edu)
} 
Table 1 Examples of target organ responses following intravenous endotoxin challenge

\begin{tabular}{|c|c|c|}
\hline End points evaluated & Results & References $^{a}$ \\
\hline \multicolumn{3}{|c|}{ Low-dose challenge (0.06-0.8 ng/kg i.v.) } \\
\hline Vital signs & Limited or no changes in heart rate, blood pressure, temperature & 14,15 \\
\hline Constitutional symptoms & No changes & 14,15 \\
\hline Neuropsychiatric symptoms & $\begin{array}{l}\text { Altered memory function, depressed mood, anhedonia, increased anxiety, altered cognitive } \\
\text { performance, variable effects on reaction time and long-term memory performance, increased } \\
\text { prefrontal cortex activation, higher glucose metabolism in the insula, and lower glucose } \\
\text { metabolism in the cingulate, enhanced pain response to capsaicin, decreased visceral sensory } \\
\text { and pain thresholds, altered subjective pain ratings }\end{array}$ & $14,16-22$ \\
\hline Blood cytokines & Increase in TNF, IL-6, IL-10, sTNFR, IL-1 ra & $14,17,23$ \\
\hline Endocrine & Variable increase in cortisol, norepinephrine & $14,18,23$ \\
\hline Hematological & Increased neutrophils and decreased lymphocytes & 23 \\
\hline \multicolumn{3}{|c|}{ High-dose challenge (1-4 ng/kg i.v.) } \\
\hline Vital signs & Fever, increased heart and respiratory rates; prolonged fever in older subjects & 24,25 \\
\hline Constitutional symptoms & Chills, rigors, malaise, myalgia, headache, nausea, vomiting (rare) & 24 \\
\hline Cardiovascular symptoms & $\begin{array}{l}\text { Increased cardiac output, decreased left ventricular function, widened pulse pressure, } \\
\text { decrease in mean arterial pressure, alterations in heart rate regularity, greater hypotension } \\
\text { in the elderly, suppressive effects on postsynaptic sympathetic nerve activity to the muscle } \\
\text { vascular bed, altered baroreflex function }\end{array}$ & $9,26-28$ \\
\hline Respiratory symptoms & $\begin{array}{l}\text { Increased respiratory frequency, decreased inspiratory time, and widened alveolar-arterial } \\
\text { oxygen tension gradient, dyspnea, increased permeability to small molecules }\end{array}$ & 29,30 \\
\hline Gastrointestinal effects & $\begin{array}{l}\text { Increased gut permeability, increased splanchnic uptake of oxygen, lactate, amino acids, and } \\
\text { free fatty acids, increased splanchnic glucose output }\end{array}$ & 31,32 \\
\hline Renal effects & $\begin{array}{l}\text { Increase of } \beta_{2} \text {-microglobulin and } N \text {-acetyl- } \beta \text {-D-glucosaminidase urine excretion rate, urinary } \\
\text { excretion of the cytosolic glutathione-S-transferase-A } 1\end{array}$ & 33,34 \\
\hline Brain effects & $\begin{array}{l}\text { Dose-related changes in sleep: low dose increased duration of deep NREM sleep whereas } \\
\text { higher doses disrupted sleep; EEG may reflect a higher state of alertness; cerebral influx of } \\
\text { phenylalanine, an abolished influx of the BCAAs leucine and isoleucine, and an ammonia- } \\
\text { independent cerebral efflux of glutamine; reduced retinal arterial vasoconstriction, and } \\
\text { reactivity of retinal RBCs flow in response to 100\% oxygen breathing; reduced cerebral blood } \\
\text { flow }\end{array}$ & $35-39$ \\
\hline
\end{tabular}

Metabolic effects Increased energy expenditure, hyperglycemia, hypoaminoacidemia, and an increase in circulating free fatty acids; increased peripheral lactate and free fatty-acid output, along with increased peripheral uptake of glucose; increased whole-body protein breakdown; increased extraction of amino acids by splanchnic bed; increased skeletal muscle proteolysis

\begin{tabular}{|c|c|c|}
\hline Endocrine effects & $\begin{array}{l}\text { Increased ACTH, cortisol, dehydroepiandrosterone, growth hormone, procalcitonin, } \\
\alpha \text {-melanocyte-stimulating hormone, ghrelin, and epinephrine }\end{array}$ & $42-45$ \\
\hline Liver & Increased CRP, serum amyloid A, inhibition of cytochrome P450 metabolism & $46-48$ \\
\hline Hematological effects & $\begin{array}{l}\text { Leukocytosis; monocytopenia; enhanced fibrinolysis; increase in tissue plasminogen activator, } \\
\alpha_{2} \text {-plasmin inhibitor-plasmin, plasminogen-activator inhibitor-1, von Willebrand antigen, } \\
\text { thrombin-antithrombin III, prothrombin fragment F1+2, and kallikrein-kinin activation; } \\
\text { increase in microparticles and cell-associated tissue factor }\end{array}$ & $49-53$ \\
\hline
\end{tabular}

Leukocyte function Gene expression, surface marker expression, suppression of T-cell function by a subset of human neutrophils, transcriptome profile neutrophils reflect extended survival and regulation of inflammatory responses, circulating heterogeneous neutrophil pool with selective priming of the respiratory burst and the release of banded neutrophils with decreased antimicrobial capacity, decreased lymphocyte numbers after 6 hours, with shift toward Th2 cytokine response

Mediators of inflammation

Multiple cytokines and chemokines are found in blood, including increases in plasma concentrations of nitric oxide metabolites (nitrite and nitrate), thiobarbituric acid-reacting substances, reactive oxygen species from neutrophils, increases in plasma free fatty acids, TNF, IL-6, MCP-1, MIF, CRP, resistin, visfatin, LBP, HMG-B1, and myoglobin concentrations

The majority of these trials were conducted using Escherichia coli O:113 endotoxin.

ACTH, adrenocorticotropic hormone; BCAA, branched-chain amino acid; CRP, C-reactive protein; EEG, electroencephalogram; HMG-B1, high-mobility group-B1 protein; IL-6, interleukin 6; IL-1 ra, interleukin 1 receptor antagonist; LBP, lipopolysaccharide-binding protein; MCP-1, monocyte chemoattractant protein-1; MIF, macrophage migration inhibition factor; NREM, non-rapid eye movement; RBC, red blood cell; sTNFR, soluble tumor necrosis factor receptor; Th2, Thelper type 2; TNF, tumor necrosis factor.

aThe references cited are examples of studies demonstrating basic responses observed after endotoxin challenge and can be found in Supplementary References online. This list is not meant to be comprehensive, and many studies from investigators who have contributed significantly to understanding human responses to endotoxin were not cited because of space limitations. 
Table 2 Examples of responses following endotoxin challenge of mucosal surfaces or dermis

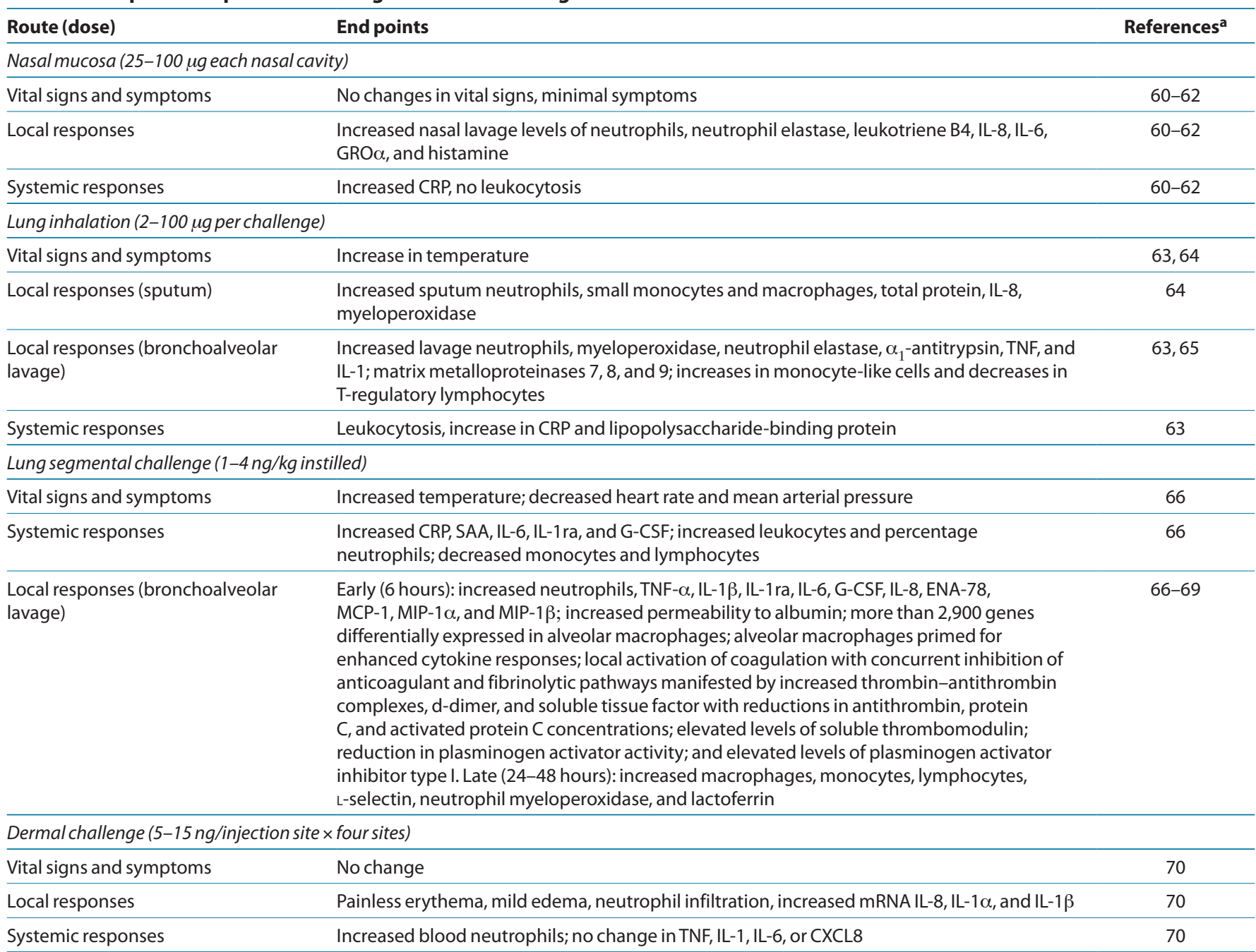

The majority of these trials were conducted using E. coli O:113 endotoxin.

CRP, C-reactive protein; CXCL8, CXC ligand 8; ENA-78, epithelial neutrophil-activating protein-78; G-CSF, granulocyte colony-stimulating factor; GRO- $\alpha$, growth-regulated peptide- $\alpha$, IL-1, interleukin 1; IL-1 ra, interleukin 1 receptor antagonist; MRNA, messenger RNA; MCP, monocyte chemotactic protein; MIP-1 $\alpha$, macrophage inflammatory protein$1 \alpha ;$ SAA, serum amyloid $A ; T N F$, tumor necrosis factor.

aThe references cited are examples of studies demonstrating basic responses observed after endotoxin challenge and can be found in Supplementary References online. This list is not meant to be comprehensive, and many studies from investigators who have contributed significantly to understanding human responses to endotoxin were not cited because of space limitations.

tions. However, endotoxin's convenience also reflects its limitations. It has doselimiting toxicities, and the short-lived response cannot reproduce sustained clinical inflammatory conditions. In addition, it cannot serve as a model of shock or a true replicating infection; rather, it can only qualitatively activate some but not all the pathways initiated by a true infection.

A safe human model of inflammation has many advantages over animal models. The latter offer rich opportunities to test key components of innate immunity by altering the dose and route of administration of challenge agents as well as genetic components of these responses. ${ }^{5}$ Manipulating these variables has often yielded mechanistic insight into inflammatory or disease processes. However, translating the relevance of these observations into human disorders requires testing them directly in a disease process or developing a human model that qualitatively reproduces these responses in humans. Often this can be achieved with endotoxin challenge studies. Furthermore, the ability to test pharmacological interventions provides an immediate link to therapies that may have broader application to human disorders. An example is eritoran, a synthetic lipid A antagonist that blocks endotoxin from binding to the MD2-TLR4 receptor. When given to volunteers before an intravenous endotoxin challenge, eritoran blocked the inflammatory effects of endotoxin. ${ }^{6}$ However, it failed to reduce mortality in patients with septic shock. ${ }^{7}$ This example confirms the challenge of extrapolating results from a successful study that addressed a single question (i.e., does eritoran alter endotoxin-associated inflammatory responses?) to application in a much more complex clinical scenario (i.e., septic shock). 
Endotoxin challenges can occur by several different routes of administration: intravenous challenge, lung inhalation or segmental instillation, nasal mucosal exposure, and intradermal challenge. The resultant inflammatory response depends on both the dose and the site of administration. Additional factors that may affect these responses include age, gender, diet, and genetics. Importantly, all of these interventions allow study of the initiation and resolution of inflammation.

A wide spectrum of systemic responses is observed after intravenous administration of $2-4 \mathrm{ng} / \mathrm{kg}$ body weight of endotoxin. ${ }^{1,8}$ Doses higher than $4 \mathrm{ng} /$ $\mathrm{kg}$ of E. coli O:113 were associated with excessive toxicities in the past and are no longer used. Following intravenous administration, endotoxin is cleared rapidly from the blood; the cascade of subsequent responses is due to activation of host innate immune responses (Table 1). Within 1 hour, subjects begin to experience varying degrees of chills, rigors, myalgia, malaise, headache, and nausea. These symptoms usually resolve within 3-5 hours. A monophasic fever curve is observed, starting within 1-2 hours, with peak temperatures rising 1 to $1.75^{\circ} \mathrm{C}$ above baseline after 3 to 4 hours and returning to baseline within 8-12 hours. In parallel, mean arterial pressure falls slightly, heart rate and respiratory rate increase, and cardiac output rises. ${ }^{9}$ Leukocytosis occurs with a predominance of neutrophil and band forms in the blood. The kallikrein-kinin, fibrinolytic, and common coagulation pathways are activated, and cytokines, chemokines, inflammatory mediators, and stress hormones are found in the circulation. Some of these responses (fever, decreased blood pressure) are accentuated when tested in subjects more than 60 years of age (Table 1). This type of challenge provides opportunities to intervene broadly (i.e., corticosteroids) or specifically (e.g., anticoagulants, anticytokines) with different agents to provide mechanistic information or proof of principle for a novel therapeutic.

Lower doses of endotoxin (0.06-0.8 $\mathrm{ng} / \mathrm{kg}$ ) by bolus or continuous infusion ${ }^{10}$ result in an increase in cytokines without changes in vital signs, briefly mimick- ing low-grade inflammatory responses (Table 1). Subjects may develop short periods of depressed mood, reduced appetite, fatigue, some cognitive impairment, and sleep disturbances, which have provided opportunities for further investigations in neuropsychiatry ${ }^{11}$ (refs 11-13 are in Supplementary References online).

Alternative routes of endotoxin exposure have been described. In contrast to the symptoms and systemic inflammatory responses observed after intravenous administration, the inhalation or instillation of endotoxin into the lungs results in limited symptoms and a local inflammatory response, characterized primarily by neutrophil airway influx that may be studied with either induced sputum or bronchoalveolar lavage. Induction of lung inflammation with inhaled endotoxin requires much larger doses $(15-50 \mu \mathrm{g})$ than the intravenous route does, because of the inefficiency of inhaled delivery. A lower-dose challenge model $(2 \mu \mathrm{g})$ using controlled inhalation was recently developed to test the antiinflammatory activity of investigational new drugs (Table 2). By contrast, direct bronchoscopic instillation of 2-4 ng/ $/ \mathrm{kg}$ into a lung segment provides a robust local inflammatory response that can be studied with bronchoalveolar lavage up to 48 hours after the challenge, allowing investigation of the initiation and resolution of inflammation in an important target organ (Table 2).

Exposure of the nasal mucosa to $50 \mu \mathrm{g}$ sprayed in each nares has no systemic effects but leads to an influx of neutrophils in nasal lavage after 6 hours (Table 2). Intradermal injection of endotoxin (10-15 ng per site of injection) results in rapid recruitment of neutrophils to the site of injection. Their presence regulates both the uptake and release of chemokines and cytokines at the site of inflammation (Table 2).

A final consideration in the use of these models of endotoxin-induced inflammation is the safety of the participants. We estimate that several thousand challenges with endotoxin by the various routes described above have been administered over the past three decades without serious adverse effects. However, some cau- tion is warranted, especially with the use of intravenous challenges; orthostatic hypotension can occur after 2-4 ng/ $\mathrm{kg}$ of endotoxin (it can be ameliorated with intravenous fluid administration). Rarely, severe vagal responses have been described that were due in part to the prechallenge hydration status of the volunteers. ${ }^{12}$ All participants in human endotoxin studies should be monitored appropriately for the specific types of challenges employed. For example, following a high-dose intravenous challenge, most investigators would monitor heart rate and blood pressure and provide maintenance fluids. Additionally, the potential interaction with any novel therapeutic agent must be assessed not only for the anti-inflammatory effects but also for the potential for exacerbated inflammation. When the interaction between endotoxin and the new intervention is not known, a dose-response evaluation should be carried out with both to ensure that no serious unexpected toxicities develop.

Endotoxin interaction with human cells remains a fundamental response in human biology. As innovative methods continue to be applied or developed to study these responses in vivo (e.g., functional magnetic resonance imaging, genomics, proteomics, metabolomics, mass cytometry, positron emission tomography), new insights into innate immune responses will be elucidated. Defining these mechanisms will then continue to provide opportunities for interventions with novel therapies in a relevant human model. ${ }^{13}$

SUPPLEMENTARY MATERIAL is linked to the online version of the paper at http://www.nature. $\mathrm{com} / \mathrm{cpt}$

\section{CONFLICT OF INTEREST}

The authors declared no conflict of interest.

\section{ACKNOWLEDGMENTS}

Supported in part by the Intramural Research Program of the Clinical Center at the National Institutes of Health (A.F.S.) and the National Center for Advancing Translational Sciences (NCATS) of the National Institutes of Health (NIH) under Award Number UL1TR001117 (R.J.N.).

\section{๑) 2014 ASCPT}

1. Bahador, M. \& Cross, A.S. From therapy to experimental model: a hundred years of endotoxin administration to human subjects. J. 
Endotoxin Res. 13, 251-279 (2007).

2. Rudbach, J.A. et al. Preparation and properties of a national reference endotoxin. J. Clin. Microbiol. 3, 21-25 (1976)

3. Poole, S. et al. (2012). Expert Committee on Specifications for Pharmaceutical Preparations: WHO International Standard for endotoxin: report of an international collaborative study to evaluate three preparations of endotoxin for their suitability to serve as the third international standard for bacterial endotoxin (WHO/BS/2012.2193 and working document QAS/12.501) World Health Organization <http:// apps.who.int/iris/bitstream/10665/96606/1/
WHO_BS_2012.2193_eng.pdf?ua $=1>$ (October 2012).

4. O'Neill, L.A., Golenbock, D. \& Bowie, A.G. The history of Toll-like receptors-redefining innate immunity. Nat. Rev. Immunol. 13, 453-460 (2013).

5. Seok, J.et al. Genomic responses in mouse models poorly mimic human inflammatory diseases. Proc. Natl. Acad. Sci. USA 110, 35073512 (2013).

6. Lynn, M. et al. Blocking of responses to endotoxin by E5564 in healthy volunteers with experimental endotoxemia. J. Infect. Dis. 187, 631-639 (2003).
7. Opal, S.M. et al. Effect of eritoran, an antagonist of MD2-TLR4, on mortality in patients with severe sepsis: the ACCESS randomized trial. JAMA 309, 1154-1162 (2013).

8. Burrell, R. Human responses to bacterial endotoxin. Circ. Shock 43, 137-153 (1994).

9. Suffredini, A.F.et al. The cardiovascular response of normal humans to the administration of endotoxin. N. Engl. J. Med. 321, 280-287 (1989).

10. Andreasen, A.S., Krabbe, K.S., Krogh-Madsen, R., Taudorf, S., Pedersen, B.K. \& Moller, K. Human endotoxemia as a model of systemic inflammation. Curr. Med. Chem. 15, 1697-1705 (2008). 